\title{
Doping Evolution of the Local Electronic and Structural Properties of the Double Perovskite $\mathrm{Ba}_{2} \mathrm{Na}_{1-x} \mathrm{Ca}_{x} \mathrm{OsO}_{6}$
}

\author{
Jagadesh Kopula Kesavan, Dario Fiore Mosca, Samuele Sanna, Francesco Borgatti, Götz Schuck, \\ Phuong Minh Tran, Patrick M. Woodward, Vesna F. Mitrović, Cesare Franchini, \\ and Federico Boscherini**
}

Cite This: J. Phys. Chem. C 2020, 124, 16577-16585

Read Online

ACCESS | 네 Metrics \& More | 回 Article Recommendations | sl Supporting Information

ABSTRACT: We present a combined experimental and computational study of the effect of charge doping in the osmium based double perovskite $\mathrm{Ba}_{2} \mathrm{Na}_{1-x} \mathrm{Ca}_{x} \mathrm{OsO}_{6}$ for $0 \leq x \leq 1$ in order to provide a structural and electronic basis for understanding this complex Dirac-Mott insulator material. Specifically, we investigate the effects of the substitution of monovalent $\mathrm{Na}$ with divalent $\mathrm{Ca}$, a form of charge doping or alloying that nominally tunes the system from $\mathrm{Os}^{7+}$ with a $5 \mathrm{~d}^{1}$ configuration to $\mathrm{Os}^{6+}$ with $5 \mathrm{~d}^{2}$ configuration. After an X-ray diffraction characterization, the local atomic and
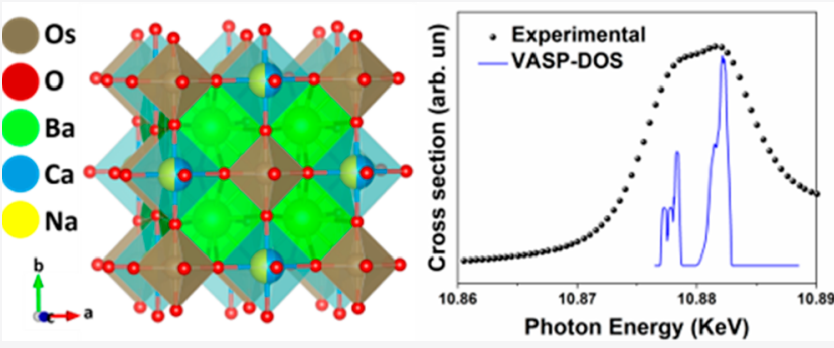
electronic structure has been experimentally probed by X-ray absorption fine structure at all the cation absorption edges at room temperature; the simulations have been performed using ab initio density functional methods. We find that the substitution of $\mathrm{Na}$ by $\mathrm{Ca}$ induces a linear volume expansion of the crystal structure which indicates an effective alloying due to the substitution process in the whole doping range. The local structure corresponds to the expected double perovskite one with rock-salt arrangement of $\mathrm{Na} / \mathrm{Ca}$ in the $\mathrm{B}$ site and Os in the $\mathrm{B}^{\prime}$ one for all the compositions. X-ray absorption near edge structure measurements show a smooth decrease of the oxidation state of Os from $7+\left(5 d^{1}\right)$ to $6+\left(5 d^{2}\right)$ with increasing $\mathrm{Ca}$ concentration, while the oxidation states of $\mathrm{Ba}, \mathrm{Na}$, and $\mathrm{Ca}$ are constant. This indicates that the substitution of $\mathrm{Na}$ by $\mathrm{Ca}$ gives rise to an effective electron transfer from the $\mathrm{B}$ to the $\mathrm{B}^{\prime}$ site. The comparison between $\mathrm{X}$-ray absorption measurements and $\mathrm{ab}$ initio simulations reveals that the expansion of the Os-O bond length induces a reduction of the crystal field splitting of unoccupied Os derived d states.

\section{INTRODUCTION}

Double perovskites (DPs) have the general formula $\mathrm{A}_{2} \mathrm{BB}^{\prime} \mathrm{O}_{6}$, with $\mathrm{A}$ an alkaline or rare earth cation and $\mathrm{B}$ and $\mathrm{B}^{\prime}$ smaller cations, often transition metals. The ideal crystal structure is similar to the more common $\mathrm{ABO}_{3}$ perovkites but involves a specific arrangement of $\mathrm{B}$ and $\mathrm{B}^{\prime}$ cations. The possible $\mathrm{B}$-cation arrangements in double perovskites ${ }^{1,2}$ include random, rocksalt, and layered while the crystal system can be cubic, rhombohedral, orthorhombic, or monoclinic, depending upon the type of cation ordering and the presence of octahedral tilting distortions. In the absence of octahedral tilting, a rocksalt cation arrangement leads to a face centered cubic unit cell in which all three lattice parameters are doubled (see inset in Figure 1).

Due to their structural and compositional flexibility, rooted in the possibility of combining $3 \mathrm{~d}, 4 \mathrm{~d}$, and $5 \mathrm{~d}$ transition metals (TMs), DPs offer a rich playground to observe several intriguing chemical and physical properties, even wider than that of standard $\mathrm{ABO}_{3}$ perovskites ${ }^{3}$ with electronic properties ranging from metallic to insulating, charge disproportionation, presence of different types of structural distortions, chemical disorder, superconductivity, ferroelectricity, and complex magnetic properties. ${ }^{4-6}$

While the structural characteristics are primarily determined by cation size mismatch, the electronic and magnetic properties are dictated by the spatial extension of the TM $\mathrm{d}$ orbitals and therefore by the balance between bandwidth, crystal-field splitting of the d-orbitals, electron-electron correlation (typically parametrized through the on-site Hubbard parameter $U$ ), and Hund's coupling constant $J$. DPs containing $3 \mathrm{~d}$ TMs tend to exhibit semiconducting behavior and local magnetic moments, whereas extended 5d orbitals favor electronic delocalization (metallicity) and magnetic fluctuations.

The presence of heavy 5d TMs brings in relatively strong spin-orbit coupling (SOC), the strength of which approaches

Received: May 28, 2020

Revised: July 8,2020

Published: July 8, 2020 


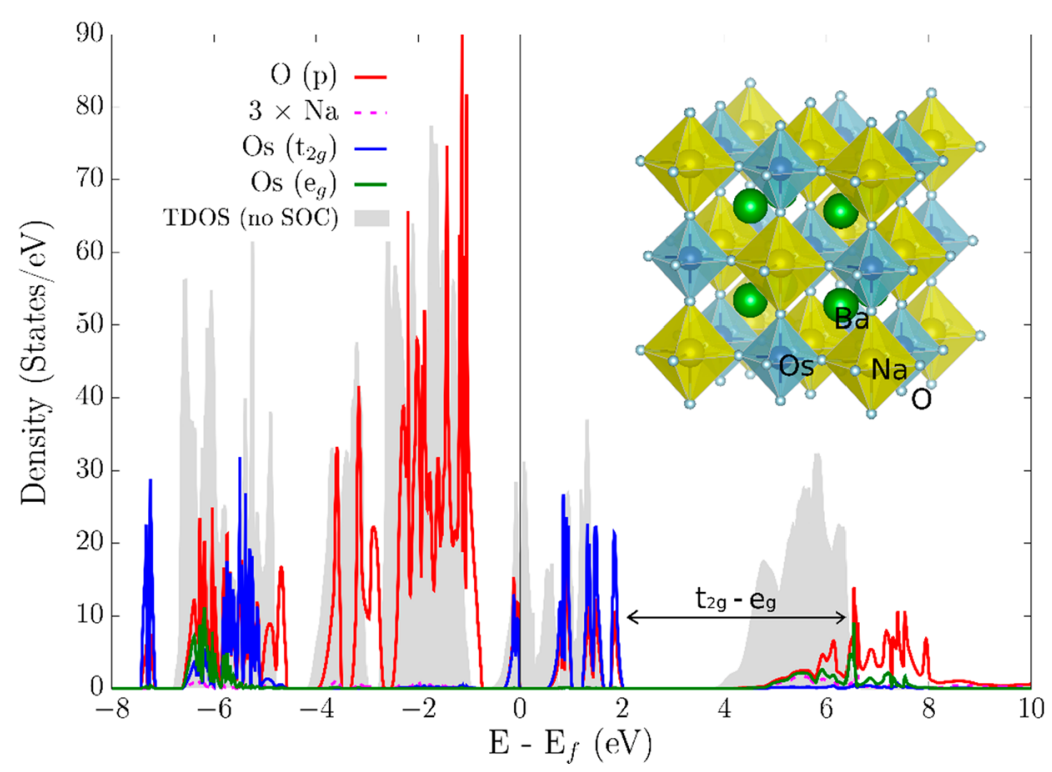

Figure 1. Basic structural and electronic characteristics of $\mathrm{Ba}_{2} \mathrm{NaOsO}_{6}$. This compound crystallizes with the rock-salt DP structure and exhibits an insulating gap between occupied and unoccupied $5 \mathrm{~d}$ Hubbard bands, due to the combined action of electronic correlation and spin-orbit coupling. In order to highlight the Dirac-Mott behavior, we have superimposed in gray the total DOS without SOC effect, which exhibits a clear metallic character.

the magnitude of crystal field splitting and electron correlation effects, making the prediction of properties more complex than for DPs containing more well-known 3d cations. Moreover, the combination of different $\mathrm{d}$-orbitals in $\mathrm{B}$ and $\mathrm{B}^{\prime}$ sites (for instance $3 \mathrm{~d} / 5 \mathrm{~d}$ ) can lead to rather exotic electronic and magnetic phases. Osmium-based perovskites and DPs represent a class of compounds in which virtually all the above-mentioned interaction channels are simultaneously active, and have attracted considerable attention in the past decade. ${ }^{4,7-13}$ In these materials the ground states are highly susceptible to external perturbations such as strain, doping, and pressure, which can drive spin-canting or insulator-to-metal transitions. $^{14-16}$

A representative material in this class is $\mathrm{Ba}_{2} \mathrm{NaOsO}_{6}$, a spinorbit coupled $5 \mathrm{~d}^{1}$ Mott insulator ${ }^{7}$ (see Figure 1) which shows a canted antiferromagnetic ground state associated with multipolar spin interactions $s^{5,17-19}$ and magnetic ordering temperature $T_{\mathrm{N}} \approx 6.3 \mathrm{~K}$. When $\mathrm{Na}$ is fully substituted by $\mathrm{Ca}$, in the resulting $5 \mathrm{~d}^{2} \mathrm{Ba}_{2} \mathrm{CaOsO}_{6}$ compound, the spin canting character is lost and the magnetic transition is greatly enhanced to $T_{\mathrm{N}} \approx 40 \mathrm{~K}^{20,21}$ Despite the signatures of collinear antiferromagnetic ordering seen in magnetic susceptibility, specific heat, and muon spin relaxation measurements, no magnetic Bragg reflections have been observed, possibly because of the small size of the ordered magnetic moment $\left(\approx 0.2 \mu_{\mathrm{B}}\right) .^{20}$ It has been proposed that the $\mathrm{Os}^{6+}$ ions in $\mathrm{Ba}_{2} \mathrm{CaOsO}_{6}$ exhibit octupolar ordering below $50 \mathrm{~K},{ }^{22,23}$ a first example of such a type of ordering in a transition metal oxide. Regardless of whether $\mathrm{Ba}_{2} \mathrm{CaOsO}_{6}$ is ultimately shown to adopt Néel or octupolar ordering, notably it remains an insulator ${ }^{20,21}$ illustrating the fact that $5 \mathrm{~d}$ double perovskites are a rare example of a robust Dirac-Mott state. This is clearly distinct from the prototypical spin-orbit coupled Mott state of $\mathrm{Sr}_{2} \mathrm{IrO}_{4}{ }^{24}$ as well as $3 \mathrm{~d}$ perovskites such as cuprates, manganites, and nickelates, in which doping causes an insulator to metal transition. ${ }^{25}$
X-ray absorption fine structure (XAFS) ${ }^{26}$ has played an important role in highlighting the local structure of perovskites and DPs. The presence and spatial distribution of antisite (AS) defects in $\mathrm{Sr}_{2} \mathrm{FeMoO}_{6}$ DPs were studied by Meneghini et $\mathrm{al}^{27}$ in this system AS defects, in the form of $\mathrm{Fe}$ on a Mo site and vice versa, are present and their concentration is known to determine the magnetoresistance ${ }^{28,29}$ and the overall electronic structure. ${ }^{30}$ The presence of strong local distortions of the Mn octahedral site in Ru based DPs was demonstrated by Bashir et al. $^{31}$

In the present paper, we focus on the structural and electronic changes induced by gradually substituting monovalent $\mathrm{Na}$ with divalent $\mathrm{Ca}$, that is, charge "doping" or "alloying", in a set of $\mathrm{Ba}_{2} \mathrm{Na}_{1-x} \mathrm{Ca}_{x} \mathrm{OsO}_{6}$ samples that span the entire compositional range $0 \leq x \leq 1$. We use a combination of $\mathrm{X}$-ray diffraction, XAFS, in both the X-ray absorption near edge structure (XANES) and extended X-ray absorption fine structure (EXAFS) regions, and ab initio density functional theory (DFT) simulations to probe the changes in the local electronic and atomic structure of all cations, with the aim of providing a structural and electronic basis for the interpretation of the physical properties.

The results show a linear volume expansion as a function of $x$, in agreement with Vegard's law. No signs of antisite disorder between $\mathrm{Os}$ and $\mathrm{Na} / \mathrm{Ca}$ are observed in X-ray powder diffraction or EXAFS data across the full doping range. A detailed analysis of the XANES and DFT data shows that the oxidation state of Os increases gradually with $\mathrm{Ca}$ concentration, showing a nominal unitary change of the Os electronic configuration from $5 \mathrm{~d}^{1}(x=0)$ to $5 \mathrm{~d}^{2}(x=1)$ in line with a formal valence picture, whereas the oxidation states of $\mathrm{Ba}, \mathrm{Ca}$, and $\mathrm{Na}$ remain essentially constant in the whole doping range. Finally, a gradual reduction of the crystal field splitting of the Os derived $\mathrm{d}$ orbitals is experimentally found with increasing $\mathrm{Ca}$ concentration. Ab initio simulations indicate that this is due to a steric effect driven by the expansion of the Os-O bonds. 
The paper is organized as follows. First, a detailed description of the experimental setup and computational procedure is presented, followed by a discussion of the structural results. Finally, we focus on the discussion of the electronic properties.

\section{EXPERIMENTAL DETAILS: SAMPLE SYNTHESIS, X-RAY DIFFRACTION CHARACTERIZATION, AND XAFS EXPERIMENTS}

Powder samples were prepared by the solid state method; stoichiometric amounts of $\mathrm{BaO}$ (Sigma-Alrich, 99.99\% trace metals basis), $\mathrm{CaO}$ (Sigma-Alrich, 99.9\% trace metals basis), $\mathrm{Na}_{2} \mathrm{O}_{2}$ (Alfa Aesar, 95\%), and Os powder (Sigma-Alrich, 99.9\% trace metals basis) were ground in a mortar and pestle, transferred to an alumina tube, and sealed in a quartz tube under vacuum. A separate alumina cap containing $\mathrm{PbO}_{2}$ was also included in the sealed quartz tube as the decomposition of $\mathrm{PbO}_{2}$ into $\mathrm{PbO}$ and $\mathrm{O}_{2}$ at $600{ }^{\circ} \mathrm{C}$ provided the oxygen source to oxidize Os metal. Because highly toxic $\mathrm{OsO}_{4}$ can form from the reaction of $\mathrm{Os}$ metal and $\mathrm{O}_{2}$ at or above $400{ }^{\circ} \mathrm{C}$, this reaction was carried out inside an evacuated silica tube and the furnace was positioned in a fume hood in case the silica tube ruptured. To ensure the full oxidation of the osmium, the amount of $\mathrm{PbO}_{2}$ was chosen to generate an excess of $1 / 4 \mathrm{~mol}$ of oxygen for every mol of the desired product. The reaction vessel was heated at $1000{ }^{\circ} \mathrm{C}$ for $24 \mathrm{~h}$. For several samples an additional step of grinding and heating for an additional $12 \mathrm{~h}$ at $1000{ }^{\circ} \mathrm{C}$ was necessary to form a homogeneous perovskite phase. In this paper we studied eight samples, the $\mathrm{Ca}$ concentration of which is reported in Table 1.

Table 1. Ca Concentration $(x)$ of the $\mathrm{Ba}_{2} \mathrm{Na}_{1-x} \mathrm{Ca}_{x} \mathrm{OsO}_{6}$ Samples

\begin{tabular}{cl} 
sample number & \multicolumn{1}{c}{$x$} \\
1 & 0 \\
2 & 0.125 \\
3 & 0.250 \\
4 & 0.375 \\
5 & 0.500 \\
6 & 0.750 \\
7 & 0.900 \\
8 & 1
\end{tabular}

Room temperature powder X-ray diffraction (PXRD) data were collected using a Bruker D8 Advance powder diffractometer $(40 \mathrm{kV}, 40 \mathrm{~mA}$, sealed $\mathrm{Cu}$ X-ray tube) equipped with an incident beam monochromator (Johansson type $\mathrm{SiO}_{2}$ crystal) and a Lynxeye XE-T position sensitive detector. Phase purity and cation ordering were determined by powder Rietveld refinement in TOPAS-Academic (version 6).

XAFS measurements were performed at the $\mathrm{K}$ edges of $\mathrm{Na}$ and $\mathrm{Ba}$, at the $\mathrm{L}_{3}$ edge of $\mathrm{Os}$, and at the $\mathrm{L}_{2,3}$ edges of $\mathrm{Ca}$, thus probing the local structure of all elements in the sample except O. All measurements reported in this paper were performed at room temperature. $\mathrm{Na} \mathrm{K}$ and $\mathrm{Ca}_{2,3}$ edge measurements were performed at the BEAR beamline ${ }^{32,33}$ of the ELETTRA facility in Trieste, Italy. Powder samples were carefully smeared on copper tape, and the XAFS spectra were measured simultaneously in total electron yield (TEY) mode and fluorescence yield (FY) mode; the former was performed by measuring the drain current to ground with a picoammeter and the latter with a $\mathrm{Ge}$ solid state detector. Os $\mathrm{L}_{3}$ edge measurements in the transmission mode were performed on the Cryo-EXAFS endstation of the KMC-3 beamline ${ }^{34}$ at the BESSY II storage ring in Berlin, Germany. Powder samples were carefully smeared on Kapton tape in several layers, the incident and transmitted photon flux were measured with ionization chambers, and a Ge sample was measured simultaneously to provide a reference for the energy scale. The $\mathrm{Ba} \mathrm{K}$ edge measurements, also in the transmission mode, were performed on the P65 beamline ${ }^{35}$ of the PETRA III storage ring in Hamburg, Germany. Samples were mixed with cellulose and pressed to form pellets, the incident and transmitted photon flux were measured with ionization chambers, and a $\mathrm{BaCO}_{4}$ sample was measured simultaneously to provide a reference for the energy scale.

\section{COMPUTATIONAL DETAILS}

All ab initio electronic structure calculations were performed in the framework of DFT using the projector augmented wave method as implemented in the Vienna ab initio simulation package (VASP)..$^{36,37}$ The exchange-correlation functional was treated at the generalized gradient approximation level following the parametrization of Perdew-Burke-Ernzerhof. ${ }^{38}$ We adopted the fully relativistic scheme (including SOC) and included an on-site Hubbard $U$ correction of $3.4 \mathrm{eV}^{17}$ for the Os d orbital using Dudarev's DFT+U approach for noncollinear spin orderings. ${ }^{14,39}$
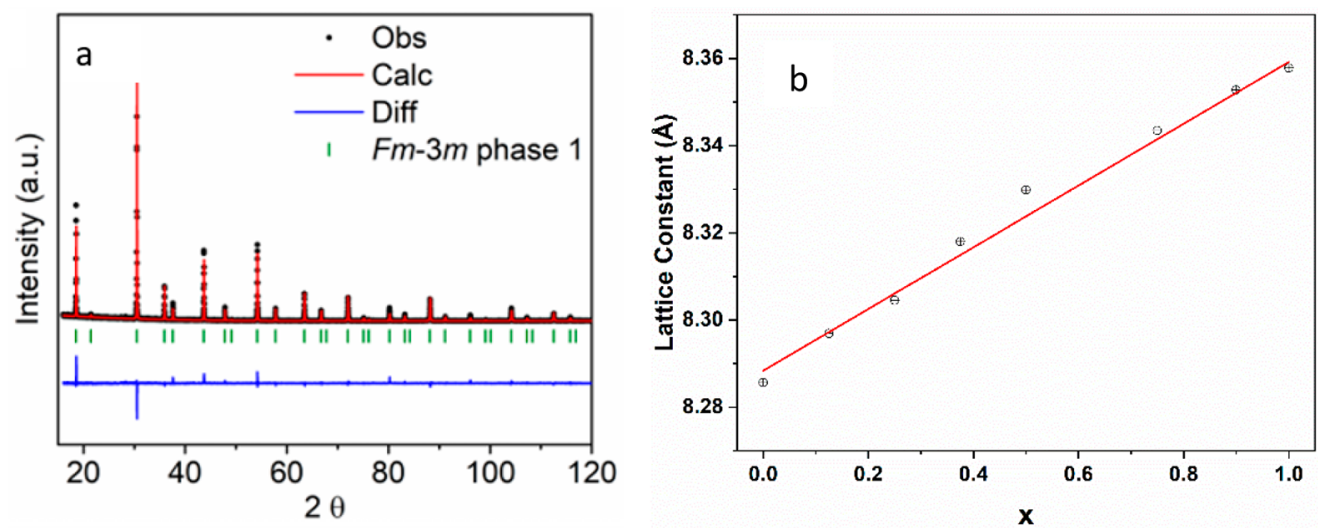

Figure 2. (a) Rietveld refinement for sample $1(x=0)$. (b) Lattice parameter versus Ca concentration. 

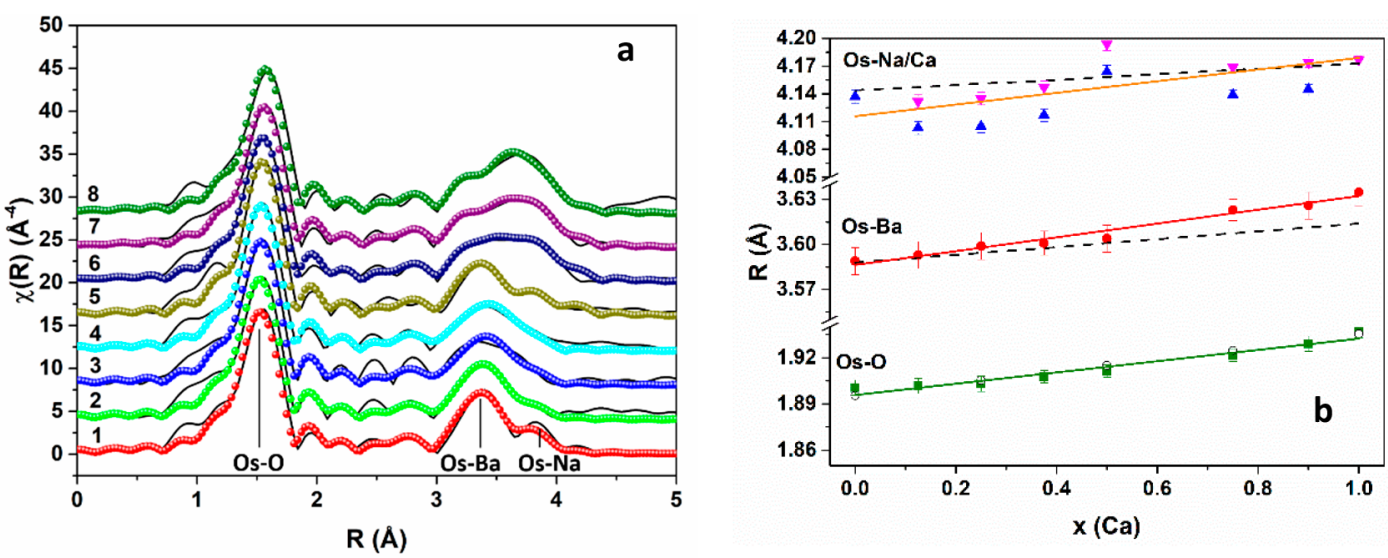

Figure 3. (a) Fourier transformed $\mathrm{Os}_{3}$ edge EXAFS spectra with their best fits. The three vertical bars at the bottom identify the single scattering contributions for $\mathrm{BaNaOsO}_{6}$. (b) Interatomic distances: green full squares, Os-O; red full circles, Os-Ba; blue full triangles, Os-Na; magenta full inverted triangles, Os-Ca. Corresponding linear fits for $\mathrm{Os}-\mathrm{O}$ and $\mathrm{Os}-\mathrm{Ba}$ are shown as green and red continuous lines. The orange line is the weighted average of the Os- $\mathrm{Ca}$ and $\mathrm{O}-\mathrm{Na}$ distances. Empty black circles are the VASP results for Os-O, and the black dashed lines are Os-Ba and $\mathrm{Os}-\mathrm{Ca} / \mathrm{Na}$ distances estimated from Vegard's law based on the XRD lattice parameters.

The $\mathrm{Ba}_{2} \mathrm{Na}_{1-x} \mathrm{Ca}_{x} \mathrm{OsO}_{6}$ double perovskite structure was modeled by a supercell containing four formula units. Starting from the $x=0$ and $x=1$ experimental structures, ${ }^{21,40}$ we have optimized the positions of $\mathrm{O}$ atoms. Charge doping induced by the $\mathrm{Ca} \rightarrow \mathrm{Na}$ chemical substitution was modeled by increasing the number of excess electrons. We have considered the following concentrations: $x=0,0.25,0.5,0.75$, and 1 . At intermediate concentrations, the lattice parameters were extracted from the experimental values obtained from the $x$ $=0$ and $x=1$ phases using Vegard's law, whereas the oxygen positions were fully relaxed at each doping level. Reciprocal space was sampled with a k-mesh of $4 \times 4 \times 4$, and an energy cutoff of $600 \mathrm{eV}$ was used for the plane wave basis set, with a convergence accuracy of $10^{-6} \mathrm{eV}$.

We adopted the following spin orderings: for $x=0$ ferromagnetically aligned spins in the (001) plane and canted between adjacent planes ${ }^{5}$ and for $x=1$ antiferromagnetically aligned spins. ${ }^{20}$ We accounted for the noncollinearity of spins by constraining the direction of the local Os magnetic moments. ${ }^{41,42}$ This is achieved in VASP by adding an additional energy term (or penalty energy) to the DFT one, parametrized by a constant parameter $\lambda$. By setting $\lambda=10$, we achieved penalty energies of $<10^{-4} \mathrm{eV}$, allowing for an overall precision of fractions of $\mathrm{meV}^{42}$

\section{RESULTS AND DISCUSSION}

X-ray Powder Diffraction. Room temperature powder Xray diffraction measurements indicate that all samples retain the cubic double perovskite structure of the end members with $F m \overline{3} m$ space group symmetry. Rietveld refinements of the crystal structure, an example of which is reported in Figure 2a, are used to extract the lattice parameters given in Table S1, revealing a steady increase with the $\mathrm{Ca}$ concentration. The linear Vegard's law relationship between cubic lattice parameter and $\mathrm{Ca}$ content, displayed in Figure $2 \mathrm{~b}$, suggests that the actual concentrations are very similar to the nominal concentrations given in Table 1 , indicating an effective alloying due to the substitution process in the whole $0 \leq x \leq 1$ doping range. No evidence for antisite disorder between $\mathrm{Os}$ and $\mathrm{Na}$ / $\mathrm{Ca}$ could be found in the refinements. The diffraction measurements also revealed small concentrations of secondary phases in some samples. In the Ca-rich samples $6(x=0.75), 7$ $(x=0.90)$, and $8(x=1)$ a $\mathrm{Ba}_{11} \mathrm{Os}_{4} \mathrm{O}_{24}$ phase ${ }^{43}$ was detected, with a mass fraction ranging from $3 \%$ to $6 \%$. Furthermore, samples $3(x=0.25)$ and $5(x=0.50)$ showed weak reflections that could be fit to a cubic double perovskite structure with a cell edge comparable to $\mathrm{Ba}_{2} \mathrm{CaOsO}_{6}(a=8.3431(2) \AA$ and mass percent $=6.6(3) \%$ for $x=0.25 ; a=8.3513(1) \AA$ and mass percent $=12.6(4) \%$ for $x=0.50)$.

Os $\mathrm{L}_{3}$ Edge EXAFS. In this section we present the evolution of the structural properties as a function of $\mathrm{Ca}$ concentration $x$ as experimentally probed by EXAFS and its interpretation aided by DFT simulations.

The Fourier transforms of the Os $\mathrm{L}_{3}$ EXAFS spectra of all samples (performed in the range $3-15 \AA^{-1}$ with a $k^{3}$ weight) are shown as continuous lines in Figure $3 \mathrm{a}$. On the basis of the structure inferred from the X-ray powder diffraction measurements, we can make the following assignments: the first peak corresponds to the Os-O first coordination shell and the features between 3 and $4.5 \AA$ are due to the Os-Ba and Os$\mathrm{Na} / \mathrm{Ca}$ contributions. The line shape of the features between 3 and $4.5 \AA$ clearly changes with composition, and there is an overall shift to longer interatomic distances with $x$, reflecting the substitution of $\mathrm{Na}$ with $\mathrm{Ca}$.

The EXAFS spectra were fitted in the range 1.2-4.2 $\AA$. using as a starting point the relaxed atomic structures calculated by VASP. The atomic coordinates were used as an input to calculate EXAFS signals using the FEFF 6.0 code. ${ }^{44}$ We found that several multiple scattering (MS) paths contribute to the EXAFS spectrum, along with the expected single scattering (SS) ones. The interatomic distances, a common energy origin shift, and Debye-Waller factors were considered as fitting parameters; the many body amplitude reduction factor $S_{0}{ }^{2}$ was determined to be 0.82 from fitting the spectrum of sample 1 ( $x$ $=0)$ and was fixed to this value for the others. The components used to fit the EXAFS spectra for this sample are listed in Table S2; for sample $8(x=1)$ Na was substituted with $\mathrm{Ca}$ and for the others a linear combination of paths involving $\mathrm{Na}$ and $\mathrm{Ca}$ was used, with a weight equal to the nominal Ca concentration. Also reported in Table S2 are expressions for the distance variation fitting parameters, many of which are geometrically related. The Debye-Waller factors for MS paths were fixed to twice the SS ones, an approximation necessary to limit the number of fitting parameters which 

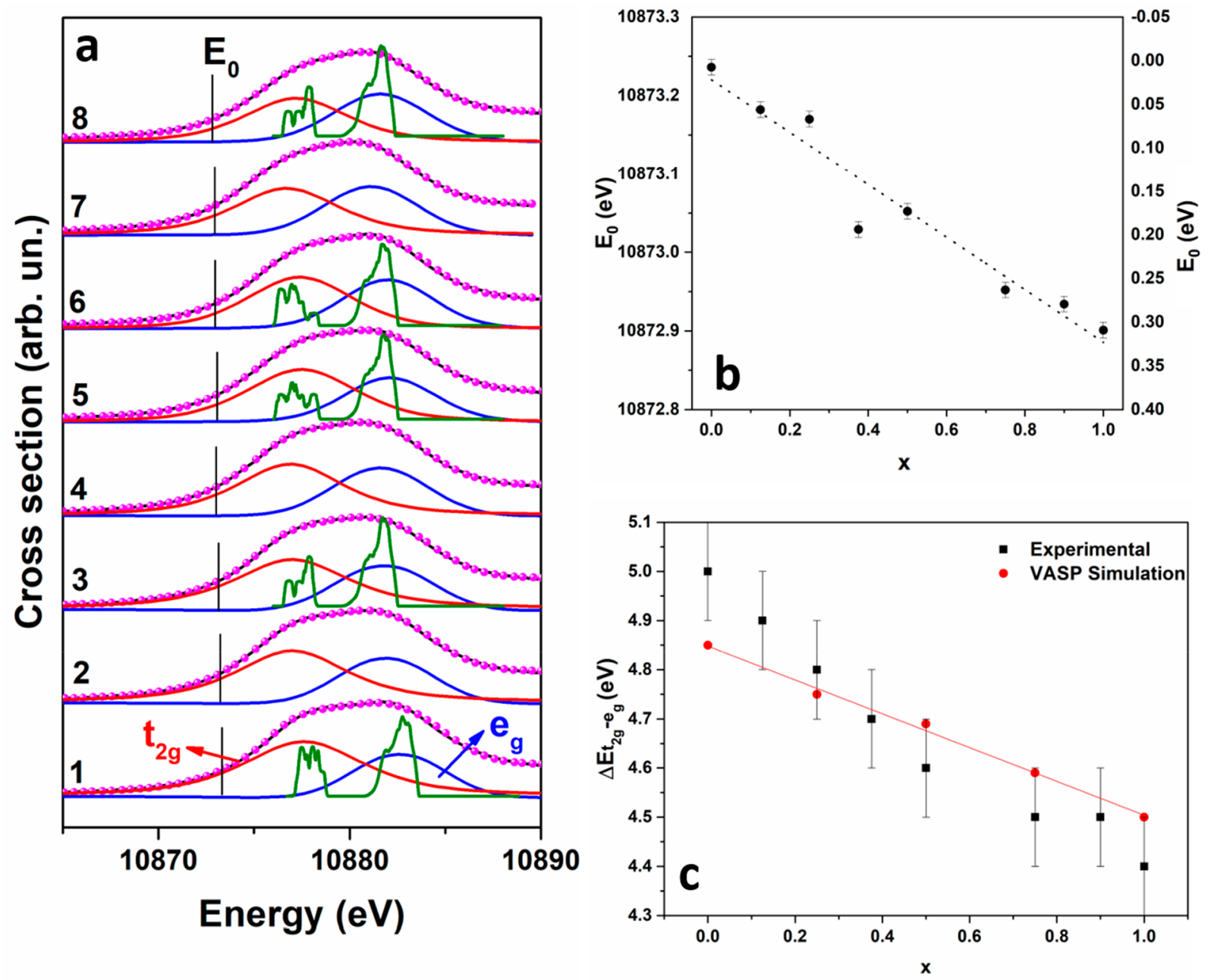

Figure 4. (a) Normalized Os $\mathrm{L}_{3}$ XANES spectra in the white line region: black line, experimental data; purple circles, fit; red and blue lines, $t_{2 g}$ and $\mathrm{e}_{\mathrm{g}}$ components, respectively; green, VASP simulations. (b) Onset $\left(E_{0}\right)$ as a function of $x$. The dotted line is a linear fit to the data. The scale on the right is the relative energy shift with respect to sample 1. (c) Comparison between the splitting between the $t_{2 g}$ and $e_{g}$ components obtained from the fit of the XANES data and from VASP.

resulted in a robust fitting; details are in Table S2. The comparison of the fits (open circles) with the experimental data is reported in Figure 3a: a very good agreement is apparent.

We found that the Os-O interatomic distances obtained from the fits to be systematically $\sim 0.022 \AA$ smaller than those deduced from the lattice parameter or the VASP simulations. We presume that this systematic effect is most likely due to small errors in the calculation of the EXAFS paths upon which the analysis is based. In order to highlight the trend in the distances as a function of $x$, we have applied a $+0.022 \AA$ correction to all the $\mathrm{Os}-\mathrm{O}$ interatomic distances determined by EXAFS. This neglects the small $(\sim 0.01 \AA)$ difference between the true interatomic distances (the difference between equilibrium atomic positions) and those determined by EXAFS, due to the perpendicular component of the thermal motion, ${ }^{26}$ which we do not expect to vary significantly with $x$. The evolution of the Os-O, Os-Ba, Os-Na and Os-Ca interatomic distances obtained from the fits is reported in Figure $3 \mathrm{~b}$ as a function of $x$; the expected linear increase of all distances with $\mathrm{Ca}$ concentration is clearly visible, even though there is some scattering for the higher distances. In the figure we also report the comparison of the EXAFS fits with estimates from VASP simulations (Os-O) and with the values deduced from Vegard's law (Os- $\mathrm{Ba}$ and $\mathrm{Os}-\mathrm{Ca} / \mathrm{Ba})$. The agreement is very reasonable. The Debye-Waller factors did not change significantly with Ca concentration and were $\sigma_{1}{ }^{2}=(3.2 \pm 0.3)$ $\times 10^{-3} \AA^{2}, \sigma_{2}{ }^{2}=(9.7 \pm 0.3) \times 10^{-3} \AA^{2}, \sigma_{3}{ }^{2}=(10 \pm 1) \times 10^{-3}$ $\AA^{2}$.

XANES. After clarifying the atomic structure, we proceed to provide a detailed analysis of the local electronic properties by combining XANES experimental data and DFT simulations. We will present XANES measurements at the $\mathrm{Os} \mathrm{L}_{3}$, Ca $\mathrm{L}_{2,3}$, $\mathrm{Ba} \mathrm{K}$, and $\mathrm{Na} \mathrm{K}$ edges and corresponding projected density of unoccupied states as derived by DFT.

As already mentioned, assuming a constant oxidation state of $\mathrm{Ba}(2+)$, the different nominal oxidation states of $\mathrm{Na}(1+)$ and $\mathrm{Ca}(2+)$ are expected to induce a variation of the oxidation state of Os from $7+$ in $\mathrm{Ba}_{2} \mathrm{NaOsO}_{6}$ to $6+$ in $\mathrm{Ba}_{2} \mathrm{CaOsO}_{6}$. This is compatible with the wide range of oxidation states of $\mathrm{Os}$ in binary compounds (from $3+$ to $8+$ ).

The Os $\mathrm{L}_{3}$ edge XANES spectra for all samples are reported in Figure S1. In Figure 4a we show the detail of the "white line" (WL) region for all samples. The presence of two components can be just appreciated, while a gradual shift toward lower 
energies of all spectral features with increasing Ca concentration is more apparent.

The position of the absorption edge, $E_{0}$, was estimated as the maximum of the first derivative, obtained by fitting with a Gaussian function using the fityk program. ${ }^{45}$ In Figure $4 \mathrm{~b}$ we report $E_{0}$ as a function of $\mathrm{x}$, with the dotted line representing a linear fit. There is a shift of the absorption edge toward lower values with increasing $x$; the overall shift from $x=0$ to $x=1$ is $\sim 0.35 \mathrm{eV}$. The shift of the X-ray absorption edge is a wellknown indication of the variation of electronic charge on the absorbing atoms, akin to the chemical shift in photoelectron spectra. Takahashi et al. ${ }^{46}$ have studied the Os $\mathrm{L}_{3}$ XANES line shape in Os metal and a series of binary compounds; they found that with increasing oxidation state there is a $\sim 0.4 \mathrm{eV}$ shift of $E_{0}$ per unit charge state. The observed variation of $E_{0}$ indicates that alloying with $\mathrm{Ca}$ induces a reduction of the charge on the Os cation of the order of 1 unit in going from $x$ $=0$ to $x=1$, as expected based on the nominal oxidation states of $\mathrm{Na}(1+), \mathrm{Ca}(2+)$, and $\mathrm{Ba}(2+)$. This indicates that an effective electron transfer from the $\mathrm{B}$ to the $\mathrm{B}^{\prime}$ site occurs as a function as a result of the of the substitution of $\mathrm{Na}$ by $\mathrm{Ca}$. Additional support for this is provided by the calculation of the Bader charge, illustrated in Figure S2, which shows that with increasing $\mathrm{Ca}$ concentration the charge on the Os site increases by approximately 0.6 electrons, while the other atomic species remain essentially unaffected (the residual excess charge flows in the interstitial region). This is solid confirmation that $\mathrm{Ca}$ acts as an electron donor in the system and that the excess charge is mainly accumulated at the Os site, illustrating the peculiarity of the $5 \mathrm{~d}$ double perovskites which preserve the Dirac-Mott insulating state ${ }^{7,21}$ despite the effective charge doping of the $\mathrm{d}$ bands.

We attribute the splitting of the WL to the effect of the crystal field, which is well-known to induce a separation of $d$ states in two $t_{2 g}$ and $e_{g}$ components in an octahedral environment. ${ }^{47,48}$ The crystal field splitting was determined by fitting the experimental data using Athena ${ }^{49}$ (version $0.9 .26)$ in a $30 \mathrm{eV}$ range centered on $E_{0}$. An arctangent and two pseudovoigt functions were used to simulate transitions to the continuum and to the $t_{2 g}$ and $e_{g}$ states, respectively. The arctangent function was centered on the value of $E_{0}$ previously found from the maximum of the first derivative and was kept fixed. The best fits and individual components are shown in Figure 4a. Also shown in the same figure are the unoccupied $d$ DOS projected on Os calculated by VASP, after a Gaussian broadening with $\sigma=0.5 \mathrm{eV}$; evidently, a good agreement in the position and relative intensity of the two components is obtained. The data show that the splitting between $t_{2 g}$ and $e_{g}$ states $\left(\Delta E_{\mathrm{t}_{2 \mathrm{~g}-\mathrm{eg}}}\right)$ decreases with increasing $x$. The energy splitting $\Delta E_{\mathrm{t}_{2 g-e g}}$ is compared to the result of VASP simulations (obtained as the difference of the center of mass of the $e_{g}$ and $t_{2 g}$ DOS) in Figure 4c; again, a good agreement is apparent. The reduction of $\Delta E_{t_{25-e g}}$ is caused by a steric effect, driven by the lengthening of the Os-O bonds, as seen in both the EXAFS analysis and VASP calculations (see Figure 3). As the $\mathrm{Os}-\mathrm{O}$ bonds lengthen in response to a gradual reduction in oxidation state from $\mathrm{Os}^{7+}$ to $\mathrm{Os}^{6+}$, the splitting between the $\mathrm{t}_{2 \mathrm{~g}}$ and $e_{g}$ orbitals is reduced. We have verified this interpretation by estimating the splitting in a hypothetical undoped phase $(x$ $=0$ ) with a volume corresponding to the fully doped end member $(x=1)$ and found that the splitting is reduced by 0.3
$\mathrm{eV}$, the same variation measured and calculated for the real $x=$ 0 and $x=1$ samples.

In Figure 5 we report the $\mathrm{Ca}_{2,3} \mathrm{XANES}$ spectra for selected samples. Spectra were recorded simultaneously in the FY and

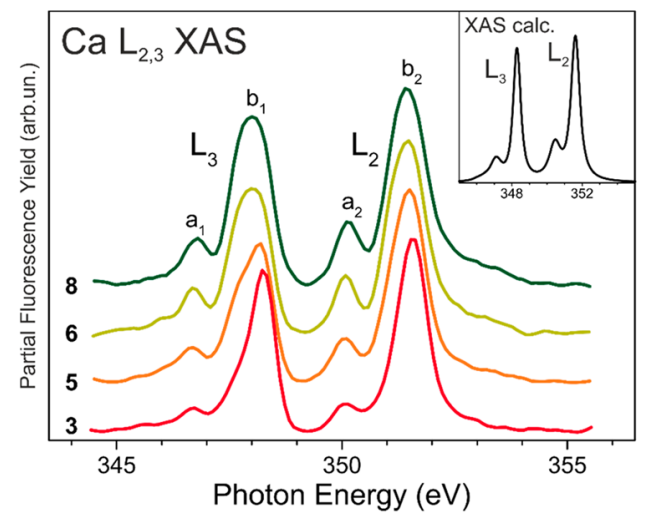

Figure 5. FY Ca $\mathrm{L}_{2,3}$ edge XANES spectra of selected samples. The spectra were normalized to the incident photon flux, background subtracted, and normalized to the maximum intensity. In the inset is shown the simulation for a $\mathrm{Ca}^{2+}$ ion in octahedral $\left(O_{h}\right)$ point group symmetry (see text for details).

TEY modes. The latter provides information related to the surface and subsurface region (about $6-8 \mathrm{~nm}$ ) of the powder grains, while the attenuation length of the former, being of the order of $100 \mathrm{~nm}$ for photon energies less than $1 \mathrm{keV}$, ensures the sensitivity to the bulk. In Figure 5 we show the FY spectra in order to be consistent with the bulk sensitivity of the other XANES measurements, while the TEY spectra are reported as Figure S3. The FY and TEY spectra are very similar except for slightly broader features in the FY spectra, which suggests a slightly greater range of similar bonding environments in the bulk of the crystalline grains. The absence of significant selfabsorption effects in the FY spectra ${ }^{50-52}$ was checked by performing measurements as a function of the grazing angle, which were found to be very similar.

The spectral features labeled $\left(a_{1}, b_{1}\right)$ and $\left(a_{2}, b_{2}\right)$ belong to the $L_{3}$ and $L_{2}$ absorption edges, respectively. The line shape of the $\mathrm{Ca} \mathrm{L}_{2,3}$ spectra is dominated by multiplet and crystal field effects. $^{53}$ In particular, the energy separation $(\sim 3.4 \mathrm{eV})$ between the $\left(a_{1}, b_{1}\right)$ and $\left(a_{2}, b_{2}\right)$ doublets is due to the spin-orbit splitting of the Ca $2 \mathrm{p}_{1 / 2}$ and $2 \mathrm{p}_{3 / 2}$ core levels while the splitting between the "a" and " $b$ " features is primarily related to the separation of the unoccupied $\mathrm{Ca} 3 \mathrm{~d}$ states into the $t_{2 g}$ and $e_{g}$ orbitals. $^{54}$

The identification of the $2+$ valence state for the $\mathrm{Ca}$ ions is supported by semiempirical multiplet calculations for the dipole transitions from the $2 \mathrm{p}^{6} 3 \mathrm{~d}^{0}$ ground state to the $2 \mathrm{p}^{5} 3 \mathrm{~d}^{1}$ final state, including crystal field effects dictated by the local octahedral coordination $\left(O_{h}\right)$ with the surrounding ligands. ${ }^{53}$ The XANES spectrum was simulated with the CTM4XAS software ${ }^{55}$ by scaling the Slater integrals by $75 \%$ in order to take into account the delocalization of the orbitals in the solid ("nephelauxetic effect") and setting the strength of the octahedral crystal field to $10 \mathrm{Dq}=1.0 \mathrm{eV}^{56}$ the result is shown in the inset of Figure 5. The experimental XANES spectrum is clearly consistent with the calculations performed for the nominal $2+$ valence state and octahedral site symmetry, thus confirming the dominant $2+$ valence state of the Ca ions. 
With increasing Ca concentration, the XANES spectra show slight broadening of the $b_{1}$ and $b_{2}$ peaks and effective shift to smaller photon energy, thus setting them closer to the $a_{1}$ and $a_{2}$ features, respectively. A contribution to this effect could be due, as for the case of Os described above, to the progressive expansion of the local structure, leading to a decrease of the crystal-field splitting. However, further effects giving rise to spectral broadening must be at play. For example, we cannot exclude the occurrence of small lattice distortions that reduce the local symmetry of the Ca lattice site, as observed for the XANES $\mathrm{L}_{2,3}$ spectra of $\mathrm{Ti}^{4+}\left(3 \mathrm{~d}^{0}\right)$ ions. ${ }^{57}$ This topic will deserve further investigation in order to clarify the leading effect. However, we notice that the physical ground state of these double perovskites is mainly governed by the Os local structure which is not affected by the possible distortions of the Ca ones.

In Figure 6 we report the $\mathrm{Na}$ K edge XANES spectra. Apart from a slight broadening for sample 3 the spectra are very

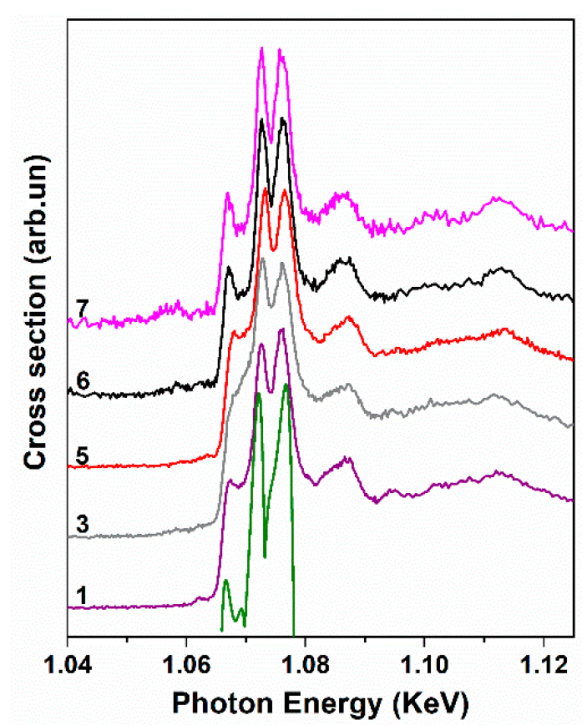

Figure 6. Continuous lines: experimental $\mathrm{Na} \mathrm{K}$ edge XANES of selected samples. Green line at the bottom: simulated $p$ DOS for $x=$ 0 .

similar. $\mathrm{Na} \mathrm{K}$ edge XANES spectra have been measured in halides, ${ }^{58}$ minerals, ${ }^{59}$ and glasses, ${ }^{60}$ but none are similar to spectra measured on our samples. To the best of our knowledge, there are no reports of spectra on perovskites containing high-Z elements such as $\mathrm{Ba}$ and Os. The similarity of all the spectra strongly suggests that the local structure and oxidation state of $\mathrm{Na}$ do not change significantly with $\mathrm{Ca}$ concentration. In Figure 6 we also report the p DOS projected on the $\mathrm{Na}$ site as calculated by VASP; to improve the comparison, the energy axis of the calculated spectra has been rescaled by a factor 0.7 , which does not affect the overall peak structure but only the bandwidth of the considered states. A good comparison is apparent, confirming that the local environment of $\mathrm{Na}$ is as expected in the double perovskite structure.

Finally, in Figure S4 we report $\mathrm{Ba} \mathrm{K}$ edge XANES measurements. The spectra are very similar, and in particular, there is no shift of position of the absorption edge, indicating a constant oxidation state on the Ba cation.

\section{CONCLUSIONS}

We have presented a joint experimental and simulation study of the evolution of the local atomic and electronic structure of $\mathrm{Ba}_{2} \mathrm{Na}_{1-x} \mathrm{Ca}_{x} \mathrm{OsO}_{6}$ double perovskites in the whole $0 \leq x \leq 1$ range, using $\mathrm{X}$-ray diffraction, $\mathrm{X}$-ray absorption spectroscopy, and $\mathrm{ab}$ initio density functional theory simulations. The data show a linear volume expansion of the crystal structure as a function of $x$, indicating an effective $\mathrm{Na}$ by $\mathrm{Ca}$ substitution process in the whole compositional range. The local structure corresponds to the expected double perovskite one with rocksalt arrangement of $\mathrm{Na}$ and $\mathrm{Ca}$ in the $\mathrm{B}$ site and $\mathrm{Os}$ in the $\mathrm{B}^{\prime}$ one. XANES measurements as a function of the $\mathrm{Ca}$ concentration show that while $\mathrm{Ba}, \mathrm{Na}$, and $\mathrm{Ca}$ display a constant oxidation state, a smooth monotonic decrease from $5 \mathrm{~d}^{1}(x=0)$ to $5 \mathrm{~d}^{2}(x=1)$ occurs for Os, in line with a formal valence picture. This remarks the peculiarity of the $5 \mathrm{~d}$ double perovskites which preserve the Dirac-Mott insulating state despite the effective charge doping of the $\mathrm{d}$ bands.

Finally, the data show a gradual reduction of the crystal field splitting of the Os derived $\mathrm{d}$ orbitals as a function of $\mathrm{Ca}$ doping. $\mathrm{Ab}$ initio simulations indicate that this is due to a steric effect driven by the expansion of the Os-O bonds with $\mathrm{Ca}$ concentration.

\section{ASSOCIATED CONTENT}

Supporting Information

The Supporting Information is available free of charge at https://pubs.acs.org/doi/10.1021/acs.jpcc.0c04807.

Os $\mathrm{L}_{3}$ edge XANES spectra in an extended energy range; plot of the Bader charge on Os; $\mathrm{Ca} \mathrm{L}_{2,3}$ edge XANES spectra recorded in the TEY mode; Ba K edge XANES spectra; lattice parameters for all samples; a description of all scattering paths used to fit the Os $\mathrm{L}_{3}$ edge EXAFS spectra (PDF)

\section{AUTHOR INFORMATION}

\section{Corresponding Author}

Federico Boscherini - Department of Physics and Astronomy, Alma Mater Studiorum-Università di Bologna, Bologna 40127, Italy; (i) orcid.org/0000-0002-9703-3903; Email: federico.boscherini@unibo.it

\section{Authors}

Jagadesh Kopula Kesavan - Department of Physics and Astronomy, Alma Mater Studiorum-Università di Bologna, Bologna 40127, Italy

Dario Fiore Mosca - Faculty of Physics, Center for Computational Materials Science, University of Vienna, Vienna 1090, Austria

Samuele Sanna - Department of Physics and Astronomy, Alma Mater Studiorum-Università di Bologna, Bologna 40127, Italy

Francesco Borgatti - Consiglio Nazionale Delle RicercheIstituto per lo Studio dei Materiali Nanostrutturati (CNRISMN), Bologna 40129, Italy; 이이.org/0000-0003-46594329

Götz Schuck - Helmholtz-Zentrum Berlin für Materialien und Energie GmbH, Berlin 14109, Germany; 이이이.org/00000002-0624-2719

Phuong Minh Tran - Department of Chemistry and Biochemistry, The Ohio State University, Columbus, Ohio 43210-1185, United States 
Patrick M. Woodward - Department of Chemistry and Biochemistry, The Ohio State University, Columbus, Ohio 43210-1185, United States; (1) orcid.org/0000-0002-34412148

Vesna F. Mitrovic - Department of Physics, Brown University, Providence, Rhode Island 02912, United States

Cesare Franchini - Department of Physics and Astronomy, Alma Mater Studiorum-Università di Bologna, Bologna 40127, Italy; Faculty of Physics, Center for Computational Materials Science, University of Vienna, Vienna 1090, Austria

Complete contact information is available at:

https://pubs.acs.org/10.1021/acs.jpcc.0c04807

\section{Author Contributions}

J.K.K. performed the XAFS experiments and the data analysis, and D.F.M. performed the DFT simulations. J.K.K. and D.F.M. contributed equally to this paper. S.S., F.Bor., and G.S. performed the XAFS experiments and made important contributions to the written text. P.M.T. and P.M.W. synthesized the samples and performed the XRD analysis. V.F.M. provided important contributions to the text. C.F. supervised the DFT simulations. F.Bos. supervised the XAFS experiments. The paper is the result of a concerted action of all authors, who have given approval to the final version of the manuscript.

\section{Funding}

C.F. acknowledges support from the Austrian Science Fund (FWF). V.F.M. acknowledges support by U.S. National Science Foundation (NSF) Grants DMR-1608760 and DMR-1905532. P.M.T. and P.M.W. acknowledge support from the Center for Emergent Materials, an NSF MRSEC, under Award DMR-1420451. J.K.K., S.S., and F.Bos. acknowledge support from the University of Bologna (RFO).

\section{Notes}

The authors declare no competing financial interest.

\section{ACKNOWLEDGMENTS}

Measurements performed at BESSY II and PETRA III have been supported by the project CALIPSOplus under Grant Agreement 730872 from the EU Framework Programme for Research and Innovation HORIZON 2020. We thank Edmund Welter for assistance in using the P65 beamline at PETRA III. We acknowledge Elettra Sincrotrone Trieste for providing access to its synchrotron radiation facilities and for financial support, and we thank Angelo Giglia for assistance in using the BEAR beamline. The computational results have been achieved using the Vienna Scientific Cluster (VSC).

\section{REFERENCES}

(1) Anderson, M. T.; Greenwood, K. B.; Taylor, G. A.; Poeppelmeier, K. R. B-Cation Arrangements in Double Perovskites. Progress in Solid State Chemistry; Pergamon, January 1, 1993; pp 197233.

(2) King, G.; Woodward, P. M. Cation Ordering in Perovskites. J. Mater. Chem. 2010, 20 (28), 5785-5796.

(3) Vasala, S.; Karppinen, M. A2B'B"O6 Perovskites: A Review. Progress in Solid State Chemistry; Elsevier Ltd., May 1, 2015; pp 1-36.

(4) Liu, M.; Hu, C.-E.; Chen, X.-R. Electronic and Magnetic Properties of 5d 1, 5d 2, and 5d 3 Double Perovskites Ba 2 MOsO 6 $(\mathrm{M}=\mathrm{K}, \mathrm{Ca}$, and $\mathrm{Sc})$ : $\mathrm{Ab}$ Initio Study. Inorg. Chem. 2018, 57 (8), 4441-4447.

(5) Lu, L.; Song, M.; Liu, W.; Reyes, A. P.; Kuhns, P.; Lee, H. O.; Fisher, I. R.; Mitrović, V. F. Magnetism and Local Symmetry Breaking in a Mott Insulator with Strong Spin Orbit Interactions. Nat. Commun. 2017, 8, 14407.

(6) Steele, A. J.; Baker, P. J.; Lancaster, T.; Pratt, F. L.; Franke, I.; Ghannadzadeh, S.; Goddard, P. A.; Hayes, W.; Prabhakaran, D.; Blundell, S. J. Low-Moment Magnetism in the Double Perovskites Ba2MOsO 6 ( $\mathrm{M}=\mathrm{Li}, \mathrm{Na})$. Phys. Rev. B: Condens. Matter Mater. Phys. 2011, 84 (14), 144416.

(7) Xiang, H. J.; Whangbo, M. H. Cooperative Effect of Electron Correlation and Spin-Orbit Coupling on the Electronic and Magnetic Properties of Ba2 NaOs O6. Phys. Rev. B: Condens. Matter Mater. Phys. 2007, 75 (5), 052407.

(8) Gangopadhyay, S.; Pickett, W. E. Interplay between Spin-Orbit Coupling and Strong Correlation Effects: Comparison of the Three Osmate Double Perovskites Ba2AOsO6 (A=Na, Ca, Y). Phys. Rev. B: Condens. Matter Mater. Phys. 2016, 93 (15), 155126.

(9) Lee, K.-W.; Pickett, W. E. Orbital-Quenching-Induced Magnetism in $\mathrm{Ba} 2 \mathrm{NaOsO6.} \mathrm{EPL} \mathrm{(Europhysics} \mathrm{Lett.} \mathrm{2007,} 80$ (3), 37008.

(10) Ahn, K. H.; Pajskr, K.; Lee, K. W.; Kuně̌, J. Calculated g -Factors of 5d Double Perovskites Ba2NaOsO6 and Ba2YOsO6. Phys. Rev. B: Condens. Matter Mater. Phys. 2017, 95 (6), 064416.

(11) Kim, B.; Liu, P.; Ergönenc, Z.; Toschi, A.; Khmelevskyi, S.; Franchini, C. Lifshitz Transition Driven by Spin Fluctuations and Spin-Orbit Renormalization in NaOsO3. Phys. Rev. B: Condens. Matter Mater. Phys. 2016, 94 (24), No. 241113(R).

(12) Hiroi, Z.; Yamaura, J.; Hirose, T.; Nagashima, I.; Okamoto, Y. Lifshitz Metal-Insulator Transition Induced by the All-in/All-out Magnetic Order in the Pyrochlore Oxide Cd2Os2O7. APL Mater. 2015, 3 (4), 041501.

(13) Calder, S.; Garlea, V. O.; McMorrow, D. F.; Lumsden, M. D.; Stone, M. B.; Lang, J. C.; Kim, J. W.; Schlueter, J. A.; Shi, Y. G.; Yamaura, K.; et al. Magnetically Driven Metal-Insulator Transition in NaOsO 3. Phys. Rev. Lett. 2012, 108 (25), 257209.

(14) Dudarev, S. L.; Botton, G. A.; Savrasov, S. Y.; Humphreys, C. J.; Sutton, A. P. Electron-Energy-Loss Spectra and the Structural Stability of Nickel Oxide: An LSDA+U Study. Phys. Rev. B: Condens. Matter Mater. Phys. 1998, 57 (3), 1505-1509.

(15) Dobrovits, S.; Kim, B.; Reticcioli, M.; Toschi, A.; Khmelevskyi, S.; Franchini, C. Doping-Induced Insulator-Metal Transition in the Lifshitz Magnetic Insulator $\mathrm{NaOsO}$ 3. J. Phys.: Condens. Matter 2019, 31 (24), 244002.

(16) Liu, P.; Reticcioli, M.; Kim, B.; Continenza, A.; Kresse, G.; Sarma, D. D.; Chen, X. Q.; Franchini, C. Electron and Hole Doping in the Relativistic Mott Insulator Sr 2 IrO 4: A First-Principles Study Using Band Unfolding Technique. Phys. Rev. B: Condens. Matter Mater. Phys. 2016, 94 (19), 195145.

(17) Erickson, A. S.; Misra, S.; Miller, G. J.; Gupta, R. R.; Schlesinger, Z.; Harrison, W. A.; Kim, J. M.; Fisher, I. R. Ferromagnetism in the Mott Insulator $\mathrm{Ba}_{2} \mathrm{NaOsO}_{6}$. Phys. Rev. Lett. 2007, 99 (1), 016404.

(18) Steele, A. J.; Baker, P. J.; Lancaster, T.; Pratt, F. L.; Franke, I.; Ghannadzadeh, S.; Goddard, P. A.; Hayes, W.; Prabhakaran, D.; Blundell, S. J. Low-Moment Magnetism in the Double Perovskites $\mathrm{Ba}_{2} \mathrm{MOsO}_{6}(M=\mathrm{Li}, \mathrm{Na})$. Phys. Rev. B: Condens. Matter Mater. Phys. 2011, 84 (14), 144416.

(19) Ishizuka, H.; Balents, L. Magnetism in $S=12$ Double Perovskites with Strong Spin-Orbit Interactions. Phys. Rev. B: Condens. Matter Mater. Phys. 2014, 90 (18), 184422.

(20) Thompson, C. M.; Carlo, J. P.; Flacau, R.; Aharen, T.; Leahy, I. A.; Pollichemi, J. R.; Munsie, T. J. S.; Medina, T.; Luke, G. M.; Munevar, J. Long-Range Magnetic Order in the $5 d^{2}$ double Perovskite $\mathrm{Ba}_{2} \mathrm{CaOsO}_{6}$ : Comparison with Spin-Disordered $\mathrm{Ba}_{2} \mathrm{YReO}_{6}$. J. Phys.: Condens. Matter 2014, 26 (30), 306003.

(21) Yamamura, K.; Wakeshima, M.; Hinatsu, Y. Structural Phase Transition and Magnetic Properties of Double Perovskites Ba2CaMO6 (M=W, Re, Os). J. Solid State Chem. 2006, 179 (3), 605-612.

(22) Maharaj, D. D.; Sala, G.; Stone, M. B.; Kermarrec, E.; Ritter, C.; Fauth, F.; Marjerrison, C. A.; Greedan, J. E.; Paramekanti, A.; Gaulin, B. D. Octupolar versus Néel Order in Cubic 5d2 Double Perovskites. Phys. Rev. Lett. 2020, 124 (8), 087206. 
(23) Paramekanti, A.; Maharaj, D. D.; Gaulin, B. D. Octupolar Order in D-Orbital Mott Insulators. Phys. Rev. B: Condens. Matter Mater. Phys. 2020, 101 (5), 054439.

(24) Jackeli, G.; Khaliullin, G. Mott Insulators in the Strong SpinOrbit Coupling Limit: From Heisenberg to a Quantum Compass and Kitaev Models. Phys. Rev. Lett. 2009, 102 (1), 017205.

(25) Imada, M.; Fujimori, A.; Tokura, Y. Metal-Insulator Transitions. Rev. Mod. Phys. 1998, 70 (4), 1039-1263.

(26) Fornasini, P. Introduction to X-Ray Absorption Spectroscopy. In Synchrotron Radiation; Springer: Berlin, 2015; pp 181-211.

(27) Meneghini, C.; Ray, S.; Liscio, F.; Bardelli, F.; Mobilio, S.; Sarma, D. D. Nature of "Disorder" in the Ordered Double Perovskite $\mathrm{Sr}_{2} \mathrm{FeMoO}_{6}$. Phys. Rev. Lett. 2009, 103 (4), 046403.

(28) Sarma, D. D.; Sampathkumaran, E. V.; Ray, S.; Nagarajan, R.; Majumdar, S.; Kumar, A.; Nalini, G.; Guru Row, T. N. Magnetoresistance in Ordered and Disordered Double Perovskite Oxide, Sr2FeMoO6. Solid State Commun. 2000, 114 (9), 465-468.

(29) García-Hernández, M.; Martínez, J. L.; Martínez-Lope, M. J.; Casais, M. T.; Alonso, J. A. Finding Universal Correlations between Cationic Disorder and Low Field Magnetoresistance in FeMo Double Perovskite Series. Phys. Rev. Lett. 2001, 86 (11), 2443-2446.

(30) Saha-Dasgupta, T.; Sarma, D. D. Ab Initio Study of Disorder Effects on the Electronic and Magnetic Structure of Sr 2 FeMoO 6. Phys. Rev. B: Condens. Matter Mater. Phys. 2001, 64 (6), 064408.

(31) Bashir, J.; Shaheen, R.; Khan, M. N. Structural Characterization of SrLaMnRuO6by Synchrotron X-Ray Powder Diffraction and X-Ray Absorption Spectroscopy. Solid State Sci. 2008, 10 (5), 638-644.

(32) Nannarone, S.; Borgatti, F.; Deluisa, A.; Doyle, B. P.; Gazzadi, G. C.; Giglia, A.; Finetti, P.; Mahne, N.; Pasquali, L.; Pedio, M.; et al. The BEAR Beamline at Elettra. In AIP Conference Proceedings; American Institute of Physics, 2004; Vol. 705, pp 450-453.

(33) Giglia, A.; Mahne, N.; Pedio, M.; Nannarone, S.; Pelizzo, M. G.; Naletto, G.; Zambolin, P. The Beam Position Monitor of the BEAR Beamline. Rev. Sci. Instrum. 2005, 76 (6), 06311.

(34) Zizak, I.; Gaal, P. The KMC-3 XPP Beamline at BESSY II. J. Large-Scale Res. Facil. JLSRF 2017, 3, 123.

(35) Welter, E.; Chernikov, R.; Herrmann, M.; Nemausat, R. A Beamline for Bulk Sample X-Ray Absorption Spectroscopy at the High Brilliance Storage Ring PETRA III. AIP Conf. Proc. 2018, 040002.

(36) Kresse, G.; Hafner, J. Ab Initio Molecular Dynamics for Liquid Metals. Phys. Rev. B: Condens. Matter Mater. Phys. 1993, 47 (1), 558561.

(37) Kresse, G.; Furthmüller, J. Efficient Iterative Schemes for Ab Initio Total-Energy Calculations Using a Plane-Wave Basis Set. Phys. Rev. B: Condens. Matter Mater. Phys. 1996, 54 (16), 11169-11186.

(38) Perdew, J. P.; Burke, K.; Ernzerhof, M. Generalized Gradient Approximation Made Simple. Phys. Rev. Lett. 1996, 77 (18), 38653868.

(39) Dudarev, S. L.; Liu, P.; Andersson, D. A.; Stanek, C. R.; Ozaki, T.; Franchini, C. Parametrization of LSDA+U for Noncollinear Magnetic Configurations: Multipolar Magnetism in UO2. Phys. Rev. Mater. 2019, 3 (8), 083802.

(40) Stitzer, K. E.; Smith, M. D.; Zur Loye, H. C. Crystal Growth of Ba2MOSO6 $(\mathrm{M}=\mathrm{Li}, \mathrm{Na})$ from Reactive Hydroxide Fluxes. Solid State Sci. 2002, 4 (3), 311-316.

(41) Hobbs, D.; Kresse, G.; Hafner, J. Fully Unconstrained Noncollinear Magnetism within the Projector Augmented-Wave Method. Phys. Rev. B: Condens. Matter Mater. Phys. 2000, 62 (17), 11556-11570.

(42) Liu, P.; Khmelevskyi, S.; Kim, B.; Marsman, M.; Li, D.; Chen, X. Q.; Sarma, D. D.; Kresse, G.; Franchini, C. Anisotropic Magnetic Couplings and Structure-Driven Canted to Collinear Transitions in Sr2IrO4 by Magnetically Constrained Noncollinear DFT. Phys. Rev. B: Condens. Matter Mater. Phys. 2015, 92 (5), 054428.

(43) Wakeshima, M.; Hinatsu, Y. Crystal Structure and Magnetic Ordering of Novel Perovskite-Related Barium Osmate Ba11Os4O24. Solid State Commun. 2005, 136 (9-10), 499-503.
(44) Rehr, J. J.; Albers, R. C. Theoretical Approaches to X-Ray Absorption Fine Structure. Rev. Mod. Phys. 2000, 72 (3), 621-654.

(45) Wojdyr, M. Fityk: A General-Purpose Peak Fitting Program. J. Appl. Crystallogr. 2010, 43 (5, Part 1), 1126-1128.

(46) Takahashi, Y.; Uruga, T.; Suzuki, K.; Tanida, H.; Terada, Y.; Hattori, K. H. An Atomic Level Study of Rhenium and Radiogenic Osmium in Molybdenite. Geochim. Cosmochim. Acta 2007, 71 (21), 5180-5190.

(47) Morrow, R.; Freeland, J. W.; Woodward, P. M. Probing the Links between Structure and Magnetism in $\mathrm{Sr}$ 2-XCaxFeOsO6 Double Perovskites. Inorg. Chem. 2014, 53 (15), 7983-7992.

(48) Kayser, P.; Injac, S.; Kennedy, B. J.; Vogt, T.; Avdeev, M.; Maynard-Casely, H. E.; Zhang, Z. Structural and Magnetic Properties of the Osmium Double Perovskites $\mathrm{Ba}_{2-x} \mathrm{Sr}_{x} \mathrm{YOsO}_{6}$. Inorg. Chem. 2017, 56 (11), 6565-6575.

(49) Ravel, B.; Newville, M. ATHENA, ARTEMIS, HEPHAESTUS: Data Analysis for X-Ray Absorption Spectroscopy Using IFEFFIT. J. Synchrotron Radiat. 2005, 12 (4), 537-541.

(50) Tröger, L.; Arvanitis, D.; Baberschke, K.; Michaelis, H.; Grimm, U.; Zschech, E. Full Correction of the Self-Absorption in SoftFluorescence Extended $\mathrm{x}$-Ray-Absorption Fine Structure. Phys. Rev. B: Condens. Matter Mater. Phys. 1992, 46 (6), 3283-3289.

(51) Carboni, R.; Giovannini, S.; Antonioli, G.; Boscherini, F. SelfAbsorption Correction Strategy for Fluorescence-Yield Soft X-ray near Edge Spectra. Phys. Scr. 2005, T115, 986.

(52) Eisebitt, S.; Böske, T.; Rubensson, J. E.; Eberhardt, W. Determination of Absorption Coefficients for Concentrated Samples by Fluorescence Detection. Phys. Rev. B: Condens. Matter Mater. Phys. 1993, 47 (21), 14103-14109.

(53) de Groot, F. M. F.; Kotani, A. Core Level Spectroscopy of Solids; Taylor and Francis, 2008.

(54) De Groot, F. M. F.; Fuggle, J. C.; Thole, B. T.; Sawatzky, G. A. L2,3 x-Ray-Absorption Edges of D0 Compounds: $\mathrm{K}+, \mathrm{Ca} 2+, \mathrm{Sc} 3+$, and Ti4+ in Oh (Octahedral) Symmetry. Phys. Rev. B: Condens. Matter Mater. Phys. 1990, 41 (2), 928-937.

(55) Ikeno, H.; de Groot, F. M. F.; Stavitski, E.; Tanaka, I. Multiplet Calculations of L 2,3 x-Ray Absorption near-Edge Structures for 3d Transition-Metal Compounds. J. Phys.: Condens. Matter 2009, 21 (10), 104208.

(56) Himpsel, F. J.; Karlsson, U. O.; McLean, A. B.; Terminello, L. J.; De Groot, F. M. F.; Abbate, M.; Fuggle, J. C.; Yarmoff, J. A.; Thole, B. T.; Sawatzky, G. A. Fine Structure of the Ca $2 p$ X-Ray-Absorption Edge for Bulk Compounds, Surfaces, and Interfaces. Phys. Rev. B: Condens. Matter Mater. Phys. 1991, 43 (9), 6899-6907.

(57) de Groot, F. M. F.; Figueiredo, M. O.; Basto, M. J.; Abbate, M.; Petersen, H.; Fuggle, J. C. 2 p X-Ray Absorption of Titanium in Minerals. Phys. Chem. Miner. 1992, 19 (3), 140-147.

(58) Murata, T.; Matsukawa, T.; Naoé, S.-i. Na K-XANES and EXAFS Studies in Sodium Halides. Phys. B 1989, 158 (1-3), 610612

(59) Neuville, D. R.; Cormier, L.; Flank, A.-M.; Prado, R. J.; Lagarde, P. Na K-Edge XANES Spectra of Minerals and Glasses. Eur. J. Mineral. 2004, 16 (5), 809-816.

(60) Ragoen, C.; Cormier, L.; Bidegaray, A. I.; Vives, S.; Henneman, F.; Trcera, N.; Godet, S. A XANES Investigation of the NetworkModifier Cations Environment before and after the $\mathrm{Na}+\mathrm{K}+$ IonExchange in Silicate Glasses. J. Non-Cryst. Solids 2018, 479, 97-104. 